\title{
Propuesta para la curricularización de las prácticas estudiantiles con enfoque territorial e interdisciplinario en la Sede Regional Chorotega, Universidad \\ Nacional
}

\section{Proposal for the Curricularization of Student Practices With a Territorial and Interdisciplinary Approach at the National University Chorotega Campus}

\author{
Sandra Lezcano Calderón \\ Universidad Nacional \\ Sede Regional Chorotega, Costa Rica \\ sandra.lezcano.calderon@una.cr
}

Recibido: 28/05/2020 Aceptado: 25/08/2020

Resumen. La Sede Regional Chorotega de la Universidad Nacional cuenta con experiencia en la articulación entre los cursos de un solo nivel (intranivel), entre niveles (internivel) y entre carreras, así como con la institucionalidad de la región y las organizaciones sociales vinculadas a esta institucionalidad para desarrollar acciones de extensión. El presente artículo tiene por objetivo presentar una propuesta metodológica para curricularizar las prácticas estudiantiles, de manera interdisciplinaria, partiendo de las necesidades del territorio en coordinación con actores locales. La propuesta se basa en la revisión del caso de la carrera de Gestión Empresarial del Turismo Sostenible de la Sede Regional Chorotega, que tiene una experiencia que aporta al tema. Así como en las características de la Sede, lo que permitió establecer las condiciones que deben cumplirse para que sea posible la curricularización. La curricularización aquí planteada establece un proceso de cuatro etapas que no son lineales, algunas se dan de manera simultánea. La primera etapa consiste en establecer la ruta formativa, lo que incluye revisiones de la experiencia en cada carrera con participación de coordinadores de carrera, académicos, estudiantes, instituciones vinculadas a las organizaciones y miembros de organizaciones para identificar aspectos a mejorar. La segunda etapa consiste en un proceso de capacitación. En la tercera etapa se 
Revista Universidad En DiÁlogo • Vol. 10, N. 2, Julio-Diciembre, 2020 • 153-166

ISSN 2215-2849 • EISSN: 2215-4752

URL: https://www.revistas.una.ac.cr/index.php/dialogo/index Correo eleCtrónico: universidadendialogo@una.ac.cr

DOI: https://doi.org/10.15359/udre.10-2.8

establecerán los mecanismos de vinculación entre carreras que permitan desarrollar un abordaje multidisciplinario. La cuarta etapa consiste en la sistematización de experiencias que permita tener lecciones aprendidas al finalizar cada año. Se concluye con una identificación de retos que deben enfrentarse para curricularizar las prácticas estudiantiles, que se vinculan con el diseño y el seguimiento de los cursos de manera concatenada internivel e intraniveles; y los costos, lo que implica disponer de recursos que permitan darle soporte a la propuesta y demanda la articulación interinstitucional para hacerla realidad.

Palabras clave: territorio, articulación interinstitucional, extensión universitaria, curricularización de la extensión, integralidad.

\begin{abstract}
The Chorotega Regional Campus of the National University of Costa Rica has experience articulating courses at a single level (intralevel), between levels (interlevel), and between careers. The campus also has experience with the region's institutions and social organizations linked to these institutions to develop extension activities. The present article aims to present a methodological proposal for the curricularization of student practices in an interdisciplinary approach, starting from the territory's needs in coordination with local stakeholders. The proposal is based on the review of a study case: the sustainable tourism management career of the Chorotega Regional Campus, whose experience contributes to the topic. The campus characteristics also allowed establishing the conditions that must be fulfilled to make possible the curriculum. The curriculum proposed here establishes a process of four stages, which are not linear, and some occur simultaneously. The first stage consists of setting the training route, which includes reviews of the experience in each career, with the participation of career coordinators, professors, students, institutions linked to organizations, and members of organizations, to identify aspects to be improved. The second stage comprises a training process. In the third stage, mechanisms will be established to link careers to develop a multidisciplinary approach. The fourth stage documents experiences that allow collecting lessons learned at the end of each year. The process concludes with the identification of challenges that must be faced to curricularize student practices linked to design and monitoring of courses in a concatenated inter- and intralevel; it also identifies costs, which implies having funds to support the proposal and requires the interinstitutional articulation to make it a reality.
\end{abstract}

Keywords: territory, interinstitutional articulation, university extension, extension curricularization, integrality.

\title{
Introducción
}

Las universidades están llamadas a fortalecer el vínculo con el entorno para responder a las demandas de la sociedad, generando oportunidades de desarrollo regional o nacional para beneficio de la comunidad, lo cual fue 
URL: https://www.revistas.una.ac.cr/index.php/dialogo/index

CoRreo electrónico: universidadendialogo@una.ac.cr

DOI: https://doi.org/10.15359/udre.10-2.8

recogido desde el año 1918 en el manifiesto del movimiento de Córdoba (Sanabria, Morales y Ortiz, 2015).

En línea con lo anterior, la Universidad Nacional de Costa Rica(UNA) desarrolla su acción sustantiva "mediante la docencia, la investigación, la extensión, la producción y otras formas que establezca la normativa institucional, las cuales se complementan y nutren mutuamente. Integra diversas prácticas y propicia el diálogo entre saberes, de manera innovadora, sistemática y transformadora" (UNA, 2015, p. 23).

Por otro lado, la UNA en su propuesta de políticas de extensión establece que:

La extensión constituye una acción sustantiva sobre la cual el modelo de universidad democrática, dialógica y sustentable, establece un compromiso social mediante la búsqueda de la calidad, pertinencia y equidad social. Dentro de este compromiso social, la extensión universitaria implica una comunicación fluida y de ambas vías con las distintas poblaciones y organizaciones, que mediante el diálogo de saberes, facilita las relaciones y representaciones con los distintos sectores de la sociedad con los que interactúa, así como con las poblaciones y los territorios; acentuando su atención en las poblaciones menos favorecidas o vulnerabilizadas socialmente. (Cerdas et al., septiembre, 2018, p. 2)

En ese sentido, la Sede Regional Chorotega de la UNA ha desarrollado una amplia experiencia en la vinculación con el entorno, respondiendo a las demandas y necesidades de diferentes sectores de la región, a través de proyectos de investigación y extensión, los cuales han incrementado su articulación con la institucionalidad a través de los años, tal como lo indican Suárez, Lezcano y Bautista (2018). Los proyectos de carácter interinstitucional son aquellos que se desarrollan en organizaciones sociales o grupos organizados, que son sujeto de atención de otra institución, y la universidad viene a aportar algún aspecto que la institucionalidad no puede abordar, es decir, hay una complementariedad.

Si bien es cierto que se ha dado una vinculación de proyectos de investigación y extensión con el entorno, la Sede ha implementado una experiencia de curricularización de las prácticas estudiantiles en la carrera de Gestión Empresarial del Turismo Sostenible que tiene como propósito contribuir con el fortalecimiento de la gestión turística sostenible en organizaciones locales, para lo cual vincula cursos de tercer nivel del I ciclo y se le da continuidad en el segundo ciclo, a través de dos cursos optativos de la carrera. Además, se vincula a estudiantes del curso de Informática y Sociedad de la carrera de Ingeniería en Sistemas. 
Revista Universidad en DiÁlogo • Vol. 10, N. 2, Julio-Diciembre, 2020 • 153-166

ISSN 2215-2849 • EISSN: 2215-4752

URL: https:/www.revistas.una.ac.cr/index.php/dialogo/index Correo eleCtrónico: universidadendialogo@una.ac.cr

DOI: https://doi.org/10.15359/udre.10-2.8

La carrera de Gestión Empresarial del Turismo Sostenible en este momento y, por lo tanto, la Sede cuentan con una experiencia que evidencia que es posible curricularizar las prácticas estudiantiles, articulándolas entre los cursos de un solo nivel (intranivel), entre niveles (internivel) y entre carreras, así como con la institucionalidad de la región y las organizaciones sociales vinculadas a esta institucionalidad.

Esta experiencia incipiente, más experiencias documentadas relacionadas con el tema, le brindan a la Sede Regional Chorotega elementos para la elaboración de una propuesta que le permita avanzar hacia la curricularización de las prácticas estudiantiles, asegurando que los jóvenes desde que inician hasta que finalizan la carrera estarán en permanente relación con el entorno, pero, además, desarrollando experiencia de trabajo con disciplinas diferentes a la suya.

Entiéndase aquí la curricularización como se plasma en las políticas institucionales de extensión universitaria de la UNA:

La integralidad de los aprendizajes en el contexto histórico, social y cultural inmediato plantea la trascendencia de articular, en docencia, la investigación y la extensión como elementos del currículo de los diferentes planes de estudio universitario, potenciando las habilidades extensionistas como formación integral para estudiantes. (Cerdas et. al., setiembre, 2018, p. 4)

En términos prácticos, al referirse a "curricularizar procesos de extensión, se debe abocar a la innovación de las prácticas estudiantiles, práctica profesional supervisada y la inclusión de cursos de extensión desde el peso en la currícula" (Cerdas et al., septiembre, 2018, p. 12).

Por otro lado, se concibe que la implementación de la curricularización de las prácticas estudiantiles debe realizarse desde el enfoque territorial de la extensión universitaria:

(...) es considerado como un conjunto integrado, integral y sistemático de acciones de extensión universitaria que buscan transformar los componentes y dinámicas determinantes de la vida y conformación de un territorio: espacial, social, económica, cultural, política, natural e históricamente delimitado, por medio de la participación y cooperación de los diversos sectores sociales que lo conforman. Para lograrlo busca la promoción y potenciación de las capacidades organizativas, políticas, económicas, culturales, sociales de las poblaciones y sectores dentro 
del espacio del territorio, buscando siempre condiciones y relaciones de inclusión, equidad, convivencia pacífica e intercultural y una gobernanza participativa y autogestionaria. (Cerdas et al., setiembre, 2018, p. 6)

La apuesta es fortalecer el proceso de formación integral de los estudiantes, que los haga personas más críticas, comprometidas con la sociedad, conocedoras de su entorno, capaces de aportar al desarrollo de un territorio, reconociendo las condiciones que este tiene. Aportando así para que los sectores menos favorecidos puedan ir avanzando en mayores capacidades, que les permitan superar sus actuales condiciones. Según Arias (2018), en Costa Rica las regiones periféricas son generadoras solo del $28 \%$ del empleo real del país, uno de cada tres empleos es no calificado y el $70 \%$ de los empleados no ha terminado la secundaria.

El presente artículo tiene por objetivo presentar una propuesta metodológica para curricularizar las prácticas estudiantiles, de manera interdisciplinaria, partiendo de las necesidades del territorio en coordinación con actores locales.

\section{Metodología}

La propuesta está basada en la revisión del resultado de la sistematización elaborada por la autora sobre la experiencia desarrollada en la carrera de Gestión Empresarial del Turismo Sostenible de la Sede Regional Chorotega, en el que se evidencian los resultados de tres años de articulación de cursos de un mismo nivel (Lezcano, 2019).

Además, se basa en las características de la Sede Regional Chorotega, la cual imparte seis carreras en un mismo espacio físico, en donde algunos académicos están en más de una carrera, así como en la experiencia de articulación interinstitucional con actores locales que la Sede ha desarrollado a través de los años. Para la construcción de la propuesta se consideraron los siguientes aspectos:

- Se reconoce que la Sede tiene un alto potencial para realizar trabajo interdisciplinario, debido a que los estudiantes de las diferentes carreras comparten un mismo espacio físico y de manera natural se comunican entre ellos. Por otro lado, la mayoría de los académicos imparten cursos en las diferentes carreras, lo que les facilita identificar potencialidades de articulación para el desarrollo de trabajos conjuntos.

- La participación de los académicos es por convencimiento, porque consideran que es pertinente y relevante para la formación de los 
estudiantes y el desarrollo del territorio. La propuesta no considera el establecimiento de una normativa que obligue a su participación, esto significa que habrá académicos que no se incorporarán y debe aceptarse de esa manera.

- Se concibe la curricularización de las prácticas estudiantiles como un proceso que inicia en el primer nivel y concluye en el último nivel de la carrera, además, debe ser concatenado de forma que lo desarrollado en el primer nivel sea la base para el siguiente nivel y así sucesivamente. Esta concatenación implica desarrollar articulaciones entre los cursos de un mismo nivel para definir qué se realizará en cada uno de ellos, pero, además, articulaciones con el siguiente nivel, por lo que, desde el inicio, se deberá conocer todo el proceso y, por lo tanto, la malla curricular.

- Las mallas de las carreras que se imparten en la Sede no fueron concebidas para curricularizar prácticas de estudiantes, por lo cual su construcción requiere un diálogo con los coordinadores de carrera, académicos y estudiantes, de tal forma que la propuesta sea construida por consenso.

- El desarrollo de la curricularización debe iniciar en cada una de las carreras de manera paralela para, posteriormente y como último paso, establecer una relación entre ellas, de tal forma que se pueda abordar la comunidad o la organización desde diferentes ámbitos, realizando un trabajo interdisciplinario.

- La participación de los estudiantes de las diferentes carreras en el proceso de curricularización de las prácticas estudiantiles interdisciplinarias es central. Algunas experiencias de la Sede evidencian que los estudiantes tienen claridad sobre cómo vincularse entre carreras para realizar trabajos conjuntos.

- El criterio de actores locales del territorio debe ser tomado en cuenta en el proceso de construcción de la propuesta. La universidad debe contribuir con los esfuerzos que otros actores realizan en el territorio dirigidos al desarrollo de comunidades, organizaciones sociales e iniciativas individuales, maximizando esfuerzos y recursos y como mecanismo que permita un mayor conocimiento de la realidad del territorio, tanto para estudiantes como para académicos. 
- La necesidad de conocer experiencias de curricularización que permitan, por un lado, motivar a los académicos a incorporarse en el proceso y, por otro lado, a brindar información y herramientas que les faciliten concebir lo que se desea realizar y la importancia de hacerlo.

- Se prevé que la implementación de la propuesta será un proceso con una duración de cuatro años, porque requiere que cada carrera desarrolle la experiencia al menos un año y luego hacer los ajustes; posteriormente, entraría el proceso de articulación entre carreras para el desarrollo de acciones interdisciplinarias.

- Aprender del proceso sobre la marcha, por lo que se propone la sistematización de experiencias como mecanismo para mejorar la curricularización de las prácticas estudiantiles.

- La necesidad de un académico que sea quien coordine todo el proceso de implementación de la curricularización de las prácticas estudiantiles en sus diferentes etapas, en conjunto con los coordinadores de carrera.

La construcción de la curricularización de las prácticas estudiantiles con enfoque territorial e interdisciplinario es un proceso que requiere la participación de todas las partes, a saber: académicos, estudiantes y actores del territorio, así como la sistematización de las experiencias para obtener lecciones aprendidas que permitan la mejora constante.

\section{Etapas de la propuesta para la curricularización de las prácticas estudiantiles}

La curricularización aquí planteada se establece como un proceso que no en todos sus tramos es lineal, hay acciones paralelas y otras son repetitivas. A continuación, se presenta un desarrollo por etapas solo para efectos de presentar la propuesta.

\section{Etapa 1: Establecimiento de la ruta formativa en cada carrera para la curricularización de las prácticas estudiantiles.}

La ruta formativa se refiere a la identificación en cada curso de cada nivel de cada carrera los temas que pueden ser aplicados en la práctica, cuyos resultados serán utilizados en el siguiente nivel, de tal manera que el estudiante desarrolle un proceso en una determinada organización o comunidad. 
Lo anterior implicará articulaciones intranivel e internivel de una carrera y requerirá el desarrollo de tres momentos: un primer momento corresponde a la identificación de posibilidades de desarrollar experiencias, iniciando con la revisión de la malla curricular por parte del coordinador de cada carrera, tal como se realizó en la carrera de Gestión del Turismo Sostenible y que se muestra en la tabla 1.

Tabla 1

Cursos y actividades en cada curso de la carrera de Gestión Empresarial del Turismo Sostenible que pueden formar parte de un proceso de curricularización de la práctica estudiantil

\begin{tabular}{|c|c|c|c|}
\hline Nivel & Ciclo & Curso & Actividades que se pueden curricularizar. \\
\hline \multirow{3}{*}{ I } & I & $\begin{array}{l}\text { Taller de Sistemas } \\
\text { Turísticos }\end{array}$ & Inventarios turísticos de zonas \\
\hline & \multirow[t]{2}{*}{ II } & $\begin{array}{l}\text { Taller de Patrimonio } \\
\text { Cultural }\end{array}$ & Identificar el patrimonio cultural \\
\hline & & Estadística Descriptiva & Generar información de comunidades \\
\hline \multirow{7}{*}{ II } & \multirow{4}{*}{ I } & $\begin{array}{l}\text { Métodos de } \\
\text { Investigación }\end{array}$ & $\begin{array}{l}\text { Identificar procesos de investigación que } \\
\text { se puedan desarrollar en las comunidades }\end{array}$ \\
\hline & & $\begin{array}{l}\text { Fundamentos de } \\
\text { Administración }\end{array}$ & $\begin{array}{l}\text { Identificar acciones de apoyo desde la } \\
\text { administración a Mipymes turísticas }\end{array}$ \\
\hline & & Contabilidad & $\begin{array}{l}\text { Identificar acciones de apoyo desde la } \\
\text { contabilidad a Mipymes turísticas }\end{array}$ \\
\hline & & Mercadeo & $\begin{array}{l}\text { Identificar acciones de apoyo desde el } \\
\text { mercadeo a Mipymes turísticas }\end{array}$ \\
\hline & \multirow{3}{*}{ II } & Hotelería & $\begin{array}{l}\text { Investigaciones cortas sobre los hoteles } \\
\text { de la región }\end{array}$ \\
\hline & & Gastronomía & $\begin{array}{l}\text { Investigaciones cortas sobre empresas } \\
\text { gastronómicas }\end{array}$ \\
\hline & & $\begin{array}{l}\text { Planificación del } \\
\text { Desarrollo }\end{array}$ & $\begin{array}{l}\text { Plan de desarrollo turístico para la región } \\
\text { (distrito, comunidad) }\end{array}$ \\
\hline
\end{tabular}


URL: https://www.revistas.una.ac.cr/index.php/dialogo/index

CoRreo electrónico: universidadendialogo@una.ac.cr

DOI: https://doi.org/10.15359/udre.10-2.8

\begin{tabular}{|c|c|c|c|}
\hline Nivel & Ciclo & Curso & Actividades que se pueden curricularizar. \\
\hline \multirow{6}{*}{ III } & \multirow{4}{*}{ I } & $\begin{array}{l}\text { Seminario Organización } \\
\text { Comunal }\end{array}$ & $\begin{array}{l}\text { Diagnóstico comunal y organizacional } \\
\text { Marco filosófico }\end{array}$ \\
\hline & & Turismo Alternativo & $\begin{array}{l}\text { Diseño de productos turísticos } \\
\text { alternativos }\end{array}$ \\
\hline & & Agencias de Viaje & $\begin{array}{l}\text { Comercialización del producto con los } \\
\text { tours operadores }\end{array}$ \\
\hline & & Recursos Humanos & Manual de puestos \\
\hline & \multirow[t]{2}{*}{ II } & $\begin{array}{l}\text { Investigación de } \\
\text { Mercado }\end{array}$ & $\begin{array}{l}\text { Determinación de la demanda real del } \\
\text { producto turístico (definido en el I ciclo } \\
\text { del III nivel) }\end{array}$ \\
\hline & & $\begin{array}{l}\text { Optativo: Comercio } \\
\text { Electrónico }\end{array}$ & $\begin{array}{l}\text { Diseño de herramientas electrónicas para } \\
\text { proyectos comunales y microempresarios }\end{array}$ \\
\hline \multirow{3}{*}{ IV } & \multirow{3}{*}{ I } & $\begin{array}{l}\text { Formulación de } \\
\text { Proyectos }\end{array}$ & $\begin{array}{l}\text { Utiliza el resultado de investigación de } \\
\text { mercado. }\end{array}$ \\
\hline & & $\begin{array}{l}\text { Optativo: Organización } \\
\text { de Actividades }\end{array}$ & $\begin{array}{l}\text { Procesos de realización de actividades o } \\
\text { eventos con organizaciones externas }\end{array}$ \\
\hline & & $\begin{array}{l}\text { Optativo: Comunicación } \\
\text { Efectiva }\end{array}$ & $\begin{array}{l}\text { Identificación del plan de medios } \\
\text { de comunicación para Mipymes y } \\
\text { organizaciones comunales }\end{array}$ \\
\hline
\end{tabular}

Nota: Elaborado por Dorado Mayorga (coordinadora de la carrera) y Lezcano Calderón (2019).

Un segundo momento corresponde a sesiones de quienes coordinan la carrera con estudiantes de tercer y cuarto nivel de cada carrera para someter a discusión la propuesta de curricularización de las prácticas estudiantiles, para que, de acuerdo con su experiencia, emitan criterio sobre ella y ayuden a identificar posibilidades de abordaje. Este proceso debe concluir con la identificación de las materias que tienen posibilidades de articulación intranivel e internivel, la metodología para realizar el proceso y un conjunto de recomendaciones para desarrollarlo. El producto será presentado a los académicos que formarán parte del proceso, quienes realizarán los aportes pertinentes para hacer los ajustes.

El tercer momento consiste en tener reuniones con actores institucionales para seleccionar a las organizaciones o comunidades en las cuales se realizarán 
Revista Universidad en DiÁlogo • Vol. 10, N. 2, Julio-Diciembre, 2020 • 153-166

ISSN 2215-2849 • EISSN: 2215-4752

URL: https://www.revistas.una.ac.cr/index.php/dialogo/index CorReo eleCtrónico: universidadendialogo@una.ac.cr

DOI: https://doi.org/10.15359/udre.10-2.8

las prácticas estudiantiles, contribuyendo con los esfuerzos que realizan. Esto fortalece el seguimiento de los procesos porque, además de los académicos, habrá otros funcionarios que también aportarán a la retroalimentación de las experiencias.

El cuarto momento corresponde al análisis de la experiencia, que se realizará con académicos y estudiantes al finalizar cada periodo de lecciones. En forma separada se harán sesiones con miembros de las organizaciones y comunidades y con funcionarios de instituciones vinculados a la experiencia para conocer su criterio con respecto a los aciertos, las dificultades encontradas y las recomendaciones de mejora que deberán ser considerados para el siguiente periodo de lecciones.

\section{Etapa 2: Desarrollar un proceso de capacitación sobre la curriculari- zación de las prácticas estudiantiles}

Como parte del fortalecimiento de la propuesta, se realizará un proceso de capacitación, el cual se desarrollará bajo diferentes modalidades. Iniciando con la realización de un simposio y un seminario sobre curricularización de las prácticas estudiantiles, dirigidos a académicos, estudiantes y actores locales con experiencia en el trabajo con las universidades, en donde se abarcarán aspectos conceptuales, experiencias de curricularización de prácticas estudiantiles en la UNA y América Latina y cursos para académicos y estudiantes que les permitan mejorar su desempeño como extensionistas.

El avance del proceso y su análisis permitirán la identificación de necesidades de capacitación para fortalecerlo, esto se hará en conjunto entre académicos y estudiantes.

\section{Etapa 3: Desarrollo de experiencias interdisciplinarias de curricula- rización de las prácticas estudiantiles}

Se partirá de la experiencia de los resultados obtenidos en la primera etapa, en la que se definió la ruta formativa de cada carrera y se identificaron aciertos, dificultades y recomendaciones de mejora. Este producto será el insumo de las sesiones con quienes coordinan las carreras, académicos y estudiantes para establecer los mecanismos de vinculación entre carreras que permitan desarrollar un abordaje interdisciplinario de la curricularización de las prácticas estudiantiles. 
Los resultados de la primera etapa, así como la propuesta de abordaje interdisciplinario de la curricularización de las prácticas estudiantiles, serán presentados a los funcionarios de las instituciones con los que se realizó la primera etapa, esto con el fin de recibir su retroalimentación, establecer los términos de la relación para continuar el trabajo conjunto con organizaciones y comunidades y elaborar un plan de acción para la implementación del proceso, el cual se compartirá con quienes coordinen las carreras y los académicos que se vincularán al proceso.

Se realizarán reuniones con los académicos, los estudiantes y con quienes coordinan las carreras al finalizar el primero y el segundo ciclos de la experiencia interdisciplinaria. Además de reuniones de seguimiento por parte de la persona responsable de coordinar el proceso, que permitan conocer avances, dificultades, aspectos de mejora y gestiones en las que haya que mediar con actores del territorio.

\section{Etapa 4: Sistematización de experiencias para la obtención de leccio- nes aprendidas}

La sistematización de las experiencias requerirá de sesiones de discusión con la participación de estudiantes, de académicos y de quienes coordinen las carreras para establecer el objeto, el objetivo y el eje de cada sistematización, con el fin de tener lecciones aprendidas sobre diferentes aristas de la experiencia al finalizar cada año.

La sistematización requerirá la elaboración de un plan de trabajo para lograr:

- Una reconstrucción histórica de las experiencias seleccionadas con participación de los involucrados, lo que requiere que quienes coordinan las carreras y los académicos lleven un registro del proceso.

- Una reflexión crítica sobre la experiencia seleccionada con participación de los involucrados

- La determinación de lecciones aprendidas con participación de los involucrados

El conjunto de sistematizaciones deberá dar insumos para construir una propuesta de curricularización de las prácticas de estudiantiles de carácter interdisciplinario para la Sede. 
Revista Universidad en DiÁlogo • Vol. 10, N. 2, Julio-Diciembre, 2020 • 153-166

ISSN 2215-2849 • EISSN: 2215-4752

URL: https:/www.revistas.una.ac.cr/index.php/dialogo/index Correo eleCtrónico: universidadendialogo@una.ac.cr

DOI: https://doi.org/10.15359/udre.10-2.8

La sistematización, tal como lo indica Cifuentes (2016):

Permite construir comprensiones y valoraciones sobre experiencias, desde la reflexión sobre interacciones con el entorno, pensamientos, instituciones y personas, que generan la posibilidad de crecer, ampliar paradigmas, preguntar desde opciones, integrar saberes, posibilitar nuevos pensamientos y renovadas prácticas, para desarrollar conciencia crítica y transformadora. (p. 9)

Por lo anterior, necesariamente en la sistematización de la experiencia debe involucrarse a los actores institucionales del territorio y de las comunidades $\mathrm{u}$ organizaciones con los que se desarrolló la experiencia, para que efectivamente haya una mayor criticidad y aprendizaje de las partes sobre lo realizado.

\section{Retos para el desarrollo del proceso de curricularización de las prácticas estudiantiles}

La curricularización de las prácticas estudiantiles innova la forma en que se ha desarrollado tradicionalmente la extensión universitaria, que ha sido en su mayoría a través de proyectos con financiamiento, cuyo número es limitado por la restricción de jornadas académicas con que cuenta la Sede, los académicos dispuestos a formularlos, los recursos para sostenerlos y donde solo algunos estudiantes se incorporan.

El proceso de integrar la extensión con la docencia, en el que se vinculan estas dos acciones sustantivas de manera sistemática y que conlleva la incorporación de la investigación, prevé algunos retos y otros saldrán a la luz sobre la marcha. Los retos que se identifican son:

En la práctica universitaria, la regla general es que cada académico piensa y desarrolla de manera individual los cursos que le corresponden, no existe una interacción entre académicos para vincular el quehacer. Esta es una propuesta de formación más integral para el estudiante durante su carrera, porque incorpora al menos dos acciones sustantivas, docencia y extensión, que los académicos deben estructurar producto de la discusión con sus pares del mismo nivel, lo cual deberá estar concatenado con los otros niveles, asegurando que los estudiantes apliquen sus conocimientos en el territorio, contribuyendo con la formación de un profesional con mayor conocimiento de su entorno, más sensible y comprometido con los sectores vulnerables.

El proceso, tal como está concebido, demanda una disposición de escuchar al otro, planificando el curso en función de un proyecto conjunto, su implemen- 
URL: https://www.revistas.una.ac.cr/index.php/dialogo/index

CoRreo electrónico: universidadendialogo@una.ac.cr

DOI: https://doi.org/10.15359/udre.10-2.8

tación no es una suma de acciones en donde cada académico valora únicamente lo que corresponde a su curso. Esto requiere pensar todo el proceso de manera integral, lo cual implica la construcción de formatos de documentos y criterios de evaluación que guiarán a los estudiantes, con la participación de los actores del territorio con los que se articula el proceso, tanto de la institucionalidad como de las organizaciones con las que se trabajará.

Esto es importante porque en el proceso no todos los estudiantes llevan las mismas materias, algunos van rezagados o simplemente por razones laborales llevan una o dos materias. ¿Cómo resolver esto en términos de desarrollo de la experiencia y la evaluación del trabajo?

Lo anterior requiere disposición de los académicos, porque implica sesiones de trabajo que demandan mucha flexibilidad para encontrar soluciones a estas realidades, establecer los horarios de encuentro para poder dar seguimiento conjunto al proceso que desarrollan los estudiantes y reuniones con los funcionarios de instituciones contrapartes de las experiencias. Este tal vez sea el mayor reto de la propuesta, la disposición a la interacción, tanto entre académicos como con los actores del territorio, pero también representa una mayor oportunidad para conocer el territorio y acercarse a más sectores vulnerables.

Un aspecto fundamental es valorar los costos, lo cuales aumentan conforme aumenta la distancia de la Sede al lugar donde se desarrolla la experiencia, especialmente si es en espacios rurales, donde el transporte público es escaso o nulo. Esto requiere que los estudiantes cuenten con recursos que les permitan pagar un transporte, alimentación y materiales de trabajo.

En el caso de los académicos, están los que manejan un vehículo institucional, pero también aquellos que, por diferentes razones, no lo hacen y requieren un chofer, lo cual es una limitante cuando se cuenta con dos en la Sede, que, además, deben responder a otras necesidades, especialmente en épocas de gira de los cursos de las diferentes carreras. Entonces, al aumentar la cantidad de actividades de extensión, hay un incremento en la demanda de salidas de vehículos.

Esto implica para la universidad disponer de recursos que permitan darle soporte a la propuesta, así como establecer acuerdos de apoyo con la institucionalidad con la cual se articula. En la medida en que no se resuelva el aspecto económico, los grupos más vulnerables que se encuentran lejos de los centros de población no se van a beneficiar de la curricularización de las prácticas estudiantiles, pues estaría determinado por la capacidad económica de los estudiantes, la cual es baja si consideramos que un porcentaje importante es becado. 
ReVista Universidad en DiÁlogo • Vol. 10, N. 2, Julio-Diciembre, 2020 • 153-166

ISSN 2215-2849 • EISSN: 2215-4752

URL: https://www.revistas.una.ac.cr/index.php/dialogo/index Correo eleCtrónico: universidadendialogo@una.ac.cr

DOI: https://doi.org/10.15359/udre.10-2.8

La Universidad Nacional en general y la Sede Regional Chorotega en particular no cuentan con procesos de formación dirigidos a estudiantes y académicos para hacer extensión, lo cual es necesario para tener claridad conceptual y herramientas básicas que permitan hacerlo adecuadamente, según la concibe la universidad.

\section{Referencias bibliográficas}

Arias, R. (9 de marzo, 2018). Transformación productiva, pobreza y desigualdad en la región Chorotega. [Diapositivas de Power Point].

Cerdas, Y., Gamboa, R., Lezcano, S., Urpí, W., León, S., Gonzales, F y Flores, M. (setiembre, 2018). Políticas institucionales de extensión universitaria. PropuestasistematizadaporlaComisión Redactora.https://drive.google. com/drive/u/0/folders/1GOniAukvu5R6dp8LOP_T6hJJ2RxI2cjI

Cifuentes, M. R. (16 al 18 de noviembre, 2016). IAP y sistematización de experiencias: apuestas, propuestas, desafíos para construir educaciones e intervenciones pertinentes y potenciadoras. VCongreso Latinoamericano de Metodología de las Ciencias Sociales (ELMeCS). Métodos, Metodologías y Nuevas Epistemologías en las Ciencias Sociales: Desafios para el Conocimiento Profundo de Nuestra América. Encuentro llevado a cabo en Mendoza FCPYS-Uncuyo. http://elmecs.fahce.unlp. edu.ar/v-elmecs/actas-2016/Cifuentes.pdf/view? searchterm=None

Lezcano, S. (2019). Propuesta metodológica para la integración de la extensión en la docencia: sistematización de una experiencia en la carrera de Turismo en la Sede Regional Chorotega, Universidad Nacional de Costa Rica. Universidad en Diálogo, 9 (2), 117-128.

Sanabria, P. E., Morales, M. E. y Ortiz, C. (2015). Interacción universidad y entorno: marco para el emprendimiento. Educ. Educ., 18(1), 111-134. 10.5294/edu.2015.18.1.7 http://web.b.ebscohost.com.una.idm.oclc.org

Suárez, A., Lezcano, S. y Bautista, B. (enero-junio, 2018). La acción sustantiva universitaria y la vinculación con el entorno: caso Sede Regional Chorotega de la Universidad Nacional de Costa Rica. Universidad en Diálogo, 8 (1), 39-56. http://dx.doi.org/10.15359/udre.8-1.3

Universidad Nacional. (2015). Estatuto Orgánico. Gaceta Extraordinaria N. ${ }^{\circ} 8$-2015. Al 20 de abril de 2015. http://www.documentos.una.ac.cr/ bitstream/handle/unadocs/6693/ESTATUTO-ORG\%C3\%81NICOUNA-digital.pdf? sequence $=1 \&$ isAllowed $=\mathrm{y}$ 